\title{
Solitons, Black Holes and Duality in String Theory
}

\author{
Ramzi R. Khuri ${ }^{\mathrm{a} *}$

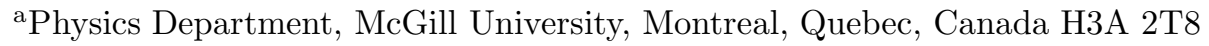

These lectures are intended as an introduction to some of the basic aspects of string solitons, duality and black holes. We begin with a discussion of the role of classical solutions in duality, then focus on string/string duality and fundamental membranes. Finally, we examine the feature of compositeness of string solitons, and its implications for bound states and black hole thermodynamics. As these lectures are aimed primarily at those less familiar with this field, technical details are minimized.

\section{INTRODUCTION}

The standard model of elementary particle physics has proven very succesful in describing three of the four fundamental forces of nature. In the most optimistic scenario, the standard model can be generalized to take the form of a grand unified theory (GUT), in which quantum chromodynamics, (QCD) describing the strong force, and the electroweak theory, unifying the weak interaction with electromagnetism, are synthesized into a single theory in which all three forces have a common origin.

The framework for studying these three forces is that of Yang-Mills gauge theory, a certain class of quantum field theory based on the principle of gauge symmetry. In any quantum-mechanical theory, the natural length scale associated with a particle of mass $m$ (such as an elementary particle) is given by the Compton wavelength $\lambda_{C}=\hbar / m c$, where $\hbar$ is Planck's constant divided by $2 \pi$ and $c$ is the speed of light. Scales less than $\lambda_{C}$ are therefore unobservable within the context of the quantum mechanics of this elementary particle.

Quantum field theory, however, has so far proven unsuccessful in describing the fourth fun-

\footnotetext{
*Based on lectures given at Asia-Pacific Center for Theoretical Physics, Seoul, Korea, 5-10 November 1996, 33rd Karpacz Winter School of Theoretical Physics, Karpacz, Poland, 13-22 February 1997 and Humboldt University of Berlin, 21-24 February 1997. To appear in Nuclear Physics B - Proc. Suppl. Research supported by NSERC of Canada and Fonds FCAR du Québec. McGill/97-4, hep-th/9704110.
}

damental force, namely gravitation. The succesful framework in this case is that of general relativity, which, however, does not seem to lend itself to a straightforward attempt at quantization. The main problem in such an endeavour is that the divergences associated with trying to quantize gravity cannot be circumvented (or "renormalized") as they are for the strong, weak and electromagnetic forces.

Among the most interesting objects predicted by general relativity are black holes, which represent the endpoint of gravitational collapes. According to relativity, an object of mass $m$ under the influence of only the gravitational force (e.g. neutral with respect to the other three forces) will collapse into a region of spacetime bounded by a surface, the event horizon, beyond which signals cannot be transmitted to an outside observer. The event horizon for the simplest case of a static, spherically symmetric black hole is located at a radius $R_{S}=2 G_{N} m / c^{2}$, the Schwarzschild radius, from the collapsed matter at the center of the sphere, where $G_{N}$ is Newton's constant.

In trying to reconcile general relativity and particle physics, even at an intuitive level, a natural question to ask is whether they have a common domain. This would arise when an elementary particle may exhibit features associated with gravitation, such as an event horizon. This may occur provided $\lambda_{C} \lesssim R_{S}$, which implies that, even within the framework of quantum mechanics, an event horizon for an elementary particle may be observable. Such a condition is equivalent 
to $m \gtrsim m_{P}=\sqrt{\hbar c / G} \sim 10^{19} \mathrm{GeV}$, the Planck mass, or $\lambda_{C} \lesssim l_{P}=\sqrt{\hbar G_{N} / c^{3}}$, the Planck scale.

It is in this domain that one may study a theory that combines quantum mechanics and gravity, the so-called quantum gravity (henceforth we use units in which $\hbar=c=1$ ).

At the present time, string theory, the theory of one-dimensional extended objects, is the only known reasonable candidate theory of quantum gravity. The divergences inherent in trying to quantize point-like gravity seem not to arise in string theory. Furthermore, string theory has the potential to unify all four fundamental forces within a common framework.

The two-dimensional worldsheet swept out by a string is embedded in a higher-dimensional (target) spacetime, which in turn represents a background for string propagation. At an intuitive level, one can see how point-like divergences may possibly be avoided in string theory by considering the four-point amplitudes arising in string theory (see, e.g., [1]). Unlike those of field theory, the four-point amplitudes in string theory do not have well-defined vertices at which the interaction can be said to take place, hence no corresponding divergences associated with the zero size of a particle. A simpler way of saying this is that the finite size of the string smooths out the divergence of the point particle.

The ground states of string theory correspond to conformal invariance of the two-dimensional sigma model of a genus zero (sphere) worldsheet. Solving the beta-function equation of this sigmamodel then correspond to classical solutions of string theory. Within this classical theory, the perturbative parameter is $\alpha^{\prime}=1 /\left(2 \pi T_{2}\right)=l_{s}^{2}$, where $T_{2}$ is the tension of the string and $l_{s}$ is the string length.

Perturbative quantum corrections in string theory take the form of an expansion in the genus of the worldsheet, with coupling parameter $g=$ $\exp \phi$, where $\phi$, the dilaton, is a dynamical scalar field.

Consistent, physically acceptable string theories possess supersymmetry between bosons and fermions, and supersymmetric string theory re- quire a ten-dimensional target space. This leads to another feature of string theory, namely, compactification, i.e., the splitting of the ten dimensional vacuum into the product of a four-dimensional vacuum and a compact sixdimensional manifold which may be shrunk to a point.

Finally, the property of string theory that is of most interest to us in these lectures is that of $d u$ ality. At the simplest level, duality is a map that takes one theory into another theory (or possibly the same theory in a different domain). An immediate consequence of duality is that the two theories are physically equivalent in the appropriate domains. It then follows that calculations performed on one side can be immediately carried over into the other, even if direct calculations in the latter theory may have previously been intractable.

The two most basic dualities in string theory are target space duality, $T$-duality, (see [2] and references therein) and strong/weak coupling duality, $S$-duality (see [3] and references therein). Suppose in a compactification one of the dimensions of the six-dimensional compactification manifold is wrapped around a circle with radius $R$. Then $T$-duality is a generalization of a map that takes a string theory with radius $R$ into a theory with radius $\alpha^{\prime} / R=l_{s}^{2} / R$. This implies that a radius smaller than the string scale is equivalent to a radius larger than the string scale. Effectively, then, the string scale is a minimal scale, which conforms to our previous intuition that the size of the string smooths out the point-like divergence. $T$-duality is a classical, worldsheet duality and in various compactifications has been shown to be an exact duality in string theory.

$S$-duality, by contrast, is a quantum (string loop), spacetime duality and generalizes the map that takes the string coupling constant $g$ to its inverse $1 / g$. Such a map takes the weak coupling domain into the strong coupling domain within a given string theory and allows us to use perturbative results in the latter. Also unlike $T$-duality, $S$-duality has only been established exactly in the low-energy limit of string theory.

These two dualities and the interplay between 
them are at the heart of the recent activity in string theory. This activity has also been fueled by the realization that perturbative string theory is insufficient to answer the most fundamental questions of string theory, such as vacuum selection, supersymmetry breaking, the cosmological constant problem and, finally, the problem of understanding quantum gravity from string theory. All these questions require nonperturbative information.

What kind of objects arise in nonperturbative physics? Solitons, or topological defects, (see [ 4 ] and references therein), are inherently nonperturbative solutions, representing objects with mass $m_{s} \sim 1 / g^{2}$, where $g$ is the coupling constant of the theory. Examples of solitons are magnetic monopoles or domain walls. The connection between duality and solitons often involves the interchange of perturbative, fundamental (electric) particles with nonperturbative, solitonic (magnetic) objects. This is the main feature of the Montonen-Olive conjecture [5] for $N=4, D=4$ supersymmetric Yang-Mills gauge theory, which postulates the existence of a dual version of the theory in which electric gauge bosons and magnetic monopoles interchange roles. In this scenario, the monopoles become the elementary particles and the gauge bosons become the solitons.

Since string theory contains various higherrank tensor fields in higher dimensions, solitons in general take the form of higher-dimensional extended objects, the so-called $p$-branes (see [6] and references therein). A $p$-brane sweeps out a $p+1$ dimensional worldvolume, generalizing the twodimensional worldsheet of the string (1-brane). In what follows, we will discuss some of the main features of these solutions of string theory and their importance in establishing dualities. At the heart of our discussion will be the connection between elementary and solitonic solutions of string theory with perturbative and nonperturbative states of string theory, a connection which has lead to many interesting results in the study of duality and black holes in string theory, but which is also still not completely understood.

In the rest of the first lecture, we summarize the elementary string and solitonic fivebrane solutions in ten dimensions, and briefly discuss string/fivebrane duality and generalizations of $p$ brane dualities. In the second lecture, we focus on the case of string/string duality and discuss the role played by duality in requiring the inclusion of a certain class of fundamental membranes (D-branes). We also comment on how $p$-branes may shed light on dualities involving eleven-dimensional supergravity. Finally, in the third lecture, we apply our knowledge of $p$-branes and duality to try to test string theory as a theory of quantum gravity. We do this by first establishing the compositeness feature of string solitons to form a bound state picture of black holes. We then combine this result with the $p$-brane/string state connection to obtain results in (classical GR) black hole thermodynamics from a microscopic counting of (quantum) string states.

\section{CLASSICAL SOLUTIONS AND DU- ALITY}

Let us consider a specific ten-dimensional superstring theory, the heterotic string theory, which is the combination of a right moving superstring and a left moving bosonic string [7]. A classical solution of heterotic string theory corresponds to superconformal invariance of the worldsheet sigma-model. The resulting beta-function equations are then equivalent to the equations of motion of an effective action, in which the worldsheet couplings $G_{M N}, B_{M N}$ and $\phi$ are interpreted as background spacetime fields, namely the metric, antisymmetric tensor and dilaton, respectively. In the low-energy limit, the tendimensional, bosonic, effective action in the gravity sector is given by

$I_{10}=\int d^{10} x \sqrt{-g}\left(R-\frac{1}{2}(\partial \phi)^{2}-\frac{1}{12} e^{-\phi} H_{3}^{2}\right)(1)$

where $g_{M N}=G_{M N} \exp (-\phi / 2)$ is the canonical (or Einstein) metric and $H_{M N P}=\partial_{[M} B_{N P]}$. This is simply the bosonic sector of $N=1$, $D=10$ supergravity.

An interesting question to pose is the following: does there exist a solution to the equations of motion of $I_{10}$ representing the exterior of a (macroscopic) straight, fundamental string? Such a solution should have a $P_{2} \times S O(8)$ symmetry, the 
$P_{2}$ representing Poincaré invariance for the string and the $S O(8)$ the maximal spatial symmetry in the rest of the space, transversal to the string.

It turns out that the answer to this question is "No". One can find a solution valid everywhere except at the origin, where the string is located. There one has to compensate a deltafunction singularity by introducing a source term, in analogy with the source charge one must introduce to account for electric charge in electrostatics: $\vec{\nabla} \cdot \vec{E}=4 \pi \rho$. In string theory, a natural source term for a string requires the addition of a sigma-model action

$$
\begin{array}{r}
S_{2}=\frac{T_{2}}{2} \int d^{2} \sigma\left(\sqrt{-\gamma} \gamma^{i j} \partial_{i} X^{M} \partial_{j} X^{N} G_{M N}\right. \\
\left.+\epsilon^{i j} \partial_{i} X^{M} \partial_{j} X^{N} B_{M N}\right),
\end{array}
$$

where $i, j$ are worldsheet indices, $M, N$ are spacetime indices and $\gamma_{i j}$ is the worldsheet metric. Then the combined action $I_{10}+S_{2}$ does indeed have the desired string-like solutions. These are given by 8

$$
\begin{aligned}
d s^{2} & =e^{3 \phi / 2}\left(-d t^{2}+d x_{1}^{2}\right)+e^{-\phi / 2} d \vec{y} \cdot d \vec{y} \\
e^{-2 \phi} & =1+k_{2} / y^{6}, \quad B_{01}= \pm e^{2 \phi},
\end{aligned}
$$

where $x_{1}$ is the direction of the string, $\vec{y}$ is a vector in the eight-dimensional transverse space (23456789) and $y$ is the radial coordinate in this space.

Notice that this solution is electrically charged under the field $B_{M N}$, which is the string-like analog to the gauge potential $A_{M}$ of an electromagnetic point particle in four dimensions. For the latter case, electric charge is given by $\int_{S^{2}} \tilde{F}_{2}$, where $S^{2}$ is a two-sphere surrounding the charge and where $\tilde{F}_{2}$, the dual of the electromagnetic field strength $F_{2}$, is given by $\tilde{F}_{M N}=$ $\frac{1}{2} \epsilon_{M N P Q} F^{P Q}$, where $\epsilon_{M N P Q}$ is the totally antisymmetric Levi-Civita tensor. For the string, the electric charge is given by $k_{2} \sim \int_{S^{7}} e^{-\phi} \tilde{H}_{3}=$ $\int_{S^{7}} K_{7}$, where $K_{7}$ is a seven-tensor, since the spacetime is ten-dimensional and the sphere surrounding the string is a seven-sphere. Also note that as we approach the singularity at $y=0$, the string coupling $g=\exp \phi \rightarrow 0$, so that this solution may reasonably correspond to the exterior solution of a perturbative string state with charge under the (axionic) field $B_{M N}$.

Now another question arises: what is the coresponding magnetic object, i.e. the one dual to the string? Again let us take the analogy with four-dimensional point particles. There the magnetic charge of a monopole is given by $\int_{S^{2}} F_{2}$, since in general $(\tilde{\tilde{F}})= \pm F$. So for the string we would expect a nonzero charge proportional to $\int_{S^{3}} H_{3}$. Such an object would then have a field strength $K_{7}$, and so would couple to a six-tensor potential $A_{6}$, where $K_{7}=d A_{6}$. An object with a six-dimensional worldwolume is called a fivebrane, since it has five spatial directions (and one time). So here we look for a solution with $P_{6} \times S O(4)$ symmetry, but consider the action $I_{10}$ alone, since we expect this object to be a soliton, hence nonsingular. This turns out to be the case, and we get the solution [9]

$d s^{2}=e^{-\phi / 2}\left(-d t^{2}+d \vec{x} \cdot d \vec{x}\right)+e^{3 \phi / 2} d \vec{y} \cdot d \vec{y}$

$e^{2 \phi}=1+k_{6} / y^{2}, \quad H_{3}= \pm 2 k_{6} \epsilon_{3}$

where here $\vec{x}$ is a vector in the five-dimensional space (12345) in which the fivebrane extends and $\vec{y}$ is a vector in the four-dimensional transverse space (6789). $\epsilon_{3}$ is the volume form on the unit three-sphere. This solution, in contrast to the string, is nonsingular, but only when written in the string sigma-model frame (with metric $\left.G_{M N}\right)$. It is also clearly nonperturbative, since as we approach $y=0$, the string coupling $g \rightarrow \infty$, and so would correspond to a nonperturbative (magnetic) state of the string theory.

We now have a fundamental object (the string) and a solitonic object (the fivebrane). Suppose we were to follow Montonen and Olive and postulate the existence of a dual theory, in which the roles of these objects were interchanged. In our original theory, heterotic string theory, the string solution is fundamental (electric), perturbative and singular, while the fivebrane solution is solitonic (magnetic), nonperturbative and nonsingular. In the dual theory, a heterotic fivebrane theory, the fivebrane is fundamental, perturbative and singu- 
lar, while the string is solitonic, nonperturbative and nonsingular.

But how can this be, since we said that the string is singular and the fivebrane nonsingular in the string sigma-model frame? In the lowenergy limit, we can rewrite the effective action $I_{10}$ in terms of $K_{7}$. However, in order to regard the fivebrane as singular, we need to couple a fivebrane source. Proceeding in analogy with the string and taking into account scaling considerations, one obtains a fivebrane sigmamodel action $S_{6}$ with six-dimensional worldvolume, with worldvolume metric $\tilde{\gamma}_{i j}$ and spacetime metric $\tilde{G}_{M N}=g_{M N} \exp (-\phi / 6)$. In terms of this metric, the fivebrane solution is indeed singular and perturbative with respect to the fivebrane coupling $\tilde{g}=\exp (-\phi / 3)$. Here one adds $S_{6}$ as a source to balance the delta-function singularities, and the charge of the fivebrane is electric with respect to the field $A_{6}$. In this frame, the string arises as a nonsingular, magnetic and nonperturbative solution.

Of course this picture is only complete in the low-energy limit, since we don't really know what the fivebrane theory is (recent progress on this issue will be discussed in other lectures later in this volume). In particular, direct quantization of the fivebrane is far from clear, and many of the nice features of strings (such as conformal invariance) are clearly absent in any naive attempt to understand fivebranes. Note also that these solutions are not solitons in the conventional sense of having a (manifestly) finite extent. A background in this case is regarded as nonsingular if the specific probe of the theory cannot reach it in finite proper time [10]. For example, the fivebrane is a nonsingular solution of string theory because a test string probe never sees a singularity at the origin, where the fivebrane is located.

As solutions, both the string and fivebrane preserve half the supersymmetries of the low-energy supergravity theory, and both can be generalized to multi-soliton solutions. In analogy with magnetic monopoles, this extension follows from the "zero-force" condition that arises as the result of the cancellation of the attractive gravitational and dilatational forces against the repul- sive antisymmetric tensor force (generalizing the electrostatic force). This condition is intimately tied to the the existence of supersymmetry, which results in the saturation of the so-called Bogomol'nyi bound [11]. In fact, both solutions may be regarded as extremal limits of two-parameter black string or black fivebrane solutions [12], in which the extremality bound is saturated so that both solutions are stable.

Duality between $p$-branes can be generalized to arbitrary higher dimensional objects in arbitrary spacetime dimemsions 13, if we put aside issues of existence and quantization of highermembrane theories. A $p$-brane with worldvolume dimension $d=p+1$ has a rank $d+1$ field strength. The dual field strength in $D$-dimensions then has rank $D-d-1$. This implies that the dual object is a $\tilde{p}$-brane with worldvolume dimension $\tilde{d}=\tilde{p}+1$ such that $\tilde{d}+1=D-d-1$. It then follows that $d+\tilde{d}+2=D$, or $p+\tilde{p}+$ $4=D$ (for string/fivebrane duality in $D=10$, $p=1$ and $\tilde{p}=5$ ). The same consequences of string/fivebrane duality, namely, fundamental/solitonic (or electric/magnetic), perturbative/nonperturbative and singular/nonsingular interchange then follow, with Dirac-type duality relations between charges, couplings and $p$-brane tensions. Both elementary and solitonic $p$-branes also in general arise as extremal limits of general black $p$-brane solutions. A feature of $p$-brane solutions that will be important to us in the third lecture is that of compositeness, i.e. the ability to construct general $p$-brane solutions as composites of the basic fundamental and solitonic building blocks.

\section{STRING/STRING DUALITY AND FUNDAMENTAL MEMBRANES}

In this lecture we focus on the case of string/string duality. One obvious reason why we would want to do so is that, since string theory is much better understood than other membrane theories, we can compare two theories we already understand that are related via duality (or a single theory in different domains). Another reason to single out string/string duality is that, in some sense, we would like a theory dual to string theory 
to have the basic features of string theory, such as conformal invariance.

String/string duality was first considered in the context of six-dimensional heterotic/heterotic duality toroidally reduced to four dimensions [14. An interesting and nontrivial consequence of this reduction is that, in the (effective) fourdimensional string/string duality, the spacetime, strong/weak coupling $S$-duality of one version goes into the worldsheet, target space $T$-duality of the dual version. Since $T$-duality is established for this type of compactification, the conjectured $S$-duality would then follow as a consequence of string/string duality.

More recently, interest has focused on duality between different string theories. In particular, the conjecture of duality was made between heterotic string theory compactified on $T_{4}$ and type IIA string theory compactified on $K 3$, a fourdimensional manifold which in the orbifold limit looks like $T_{4} / Z_{2}$ [15,16]. In this case, one has in some sense a more straightforward strong/weak coupling duality relating the two theories with $g_{\text {IIA }}=1 / g_{\text {het }}$. Evidence for this duality can be seen from considerations of low-energy fieldcontent, supersymmetry, spectra of states and even one-loop tests. Finally, from the point of view of $p$-brane solutions, the usual interchange of fundamental and solitonic solutions (both strings in this case) occurs. In particular, the fundamental heterotic string appears as a soliton of the type $I I A$ theory and vice-versa [17].

Consider now an arbitary $p$-brane solution of either of these dual theories. Let us study the behaviour of this $p$-brane under the duality map. If the $p$-brane is singular in both theories, we may discard it as unphysical. If it is nonsingular (solitonic) in both theories, then it can be included in both solitonic spectra. However, if the $p$-brane is singular in one version and nonsingular in the other, then it can neither be ignored as unphysical nor simply included in a soliton spectrum. In that case, the $p$-brane must be regarded as a fundamental source. This is the case, of course, for each of the fundamental strings (heterotic and type IIA). As it turns out, however, there also exist membrane solutions which are nonsingular in heterotic string theory but singular in type IIA
[18]. What is the interpretation of such solutions? Another puzzle lies in the peculiar $1 / g$ dependence of the mass of these membranes, intermediate between the coupling-independent mass behaviour of fundamental $p$-branes and the $1 / g^{2}$ mass behaviour of solitonic $p$-branes, the latter being typical solitonic behaviour in field theory.

The existence of these membranes, coupled with the assumption of duality, has pointed to a gap in the formulation of IIA string theory, since it appears we must add fundamental membranes to the spectrum. This puzzle was resolved in 19], where it was shown that Dirichletbranes, or D-branes, extended objects defined by mixed Dirichlet-Neumann boundary conditions for open strings, should be coupled to types IIA, IIB and type I (open string) theories and identified as states carrying both electric and magnetic Ramond-Ramond (RR) charge. As D-branes will be extensively covered later in this volume (see also 20] and references therein for a review on Dbranes), we will not discuss them in detail here. Suffice to say that this discovery solved two problems at once: the inclusion of RR states in type II string theory, previously unaccounted for in the perturbative spectrum, and the interpretation of RR charged solitons arising in the context of string duality. The mass of a D-brane state (or a $\mathrm{D} p$-brane solution) scales as $1 / g$

A picture then emerges in which fundamental and solitonic $p$-branes carrying Neveu-SchwarzNeveu-Schwarz (NSNS) charge correspond to perturbative and nonperturbative BPS states of string theory, while $p$-branes carrying RR charge correspond to D-brane BPS states which must be coupled to the perturbative spectrum.

Since type IIA supergravity is the low-energy limit of IIA string theory, and also arises as the Kaluza-Klein reduction of eleven-dimensional supergravity, a natural question to ask is whether the latter is the low-energy limit of a fundamental eleven-dimensional theory, the so-called $M$-theory [16,21]. The eventual construction of M-theory should lead to the establishment of the various string/string dualities. In this framework, the five seemingly distinct string theories arise as weak coupling limits of the various compactifications of the eleven-dimensional M-theory. 
While we still do not know exactly what Mtheory is (recent progress in this endeavour will be discussed in other lectures in this volume), we know that M-theory contains membranes and fivebranes. From the point of view of $p$-brane solutions, these are represented by a fundamental membrane [22] and a solitonic fivebrane [23]. In compactifying to lower dimensions, the membrane and fivebrane are wrapped or simply reduced to yield the various $p$-branes carrying both NSNS and RR charge. For example, in the Kaluza-Klein (KK) reduction from eleven to ten dimensions (IIA supergravity), we obtain the NSNS fundamental string (and then dualize to obtain the fivebrane) by wrapping the membrane around the KK direction, while we obtain the RR membrane in ten dimensions by simply reducing the membrane and the $R R$ point particle from the KK reduction of the metric.

Let us now look at an example of how $p$-branes can shed light on a duality involving M-theory. Following [24], let us consider the conjecture that M-theory compactified on a particular CalabiYau threefold is dual to heterotic string theory compactified on $K 3 \times S^{1}$.

Point-like (electric) states are obtained in $D=$ 5 by wrapping the membrane from M-theory around two-cycles in the Calabi-Yau space. Denote two-cycles and four-cycles respectively by $C^{2 \Lambda}$ and $C_{4 \Lambda}$, where $\Lambda=1, \ldots, h_{(1,1)}$. The charges of these states are obtained from the charge of the membrane by

$e_{\Lambda}=\int_{C_{4 \Lambda} \times S^{3}} G_{7}$,

where $G_{7}=\delta \mathcal{L} / \delta F_{4}$, where $F_{4}=d A_{3}$ is the field strength of the three-form antisymmetric tensor field.

String-like (magnetic) states in $D=5$ arise by wrapping the fivebrane around four-cycles in the Calabi-Yau space. The charges of these states are then obtained from the charge of the fivebrane by

$m_{\Lambda}=\int_{C^{2 \Lambda} \times S^{2}} F_{4}$.

Since the membrane and fivebrane are electric/magnetic duals in eleven dimensions, the above point-like and string-like states are dual to each other in the electric/magnetic sense and correspond to point-like and string-like soliton solutions. For the specific Calabi-Yau manifold $X_{24}(1,1,2,8,12)$ with $h_{(1,1)}=3$ and $h_{(2,1)}=$ 243 , we match these point and string solutions/states with perturbative and nonperturbative solutions/states of heterotic string theory compactified on $K 3 \times S^{1}$, in which the gauge symmetry is completely Higgsed.

From the ten-dimensional point of view, the heterotic string compactified on $K 3 \times S^{1}$ has the perturbative fundamental string state with charge

$\tilde{m}_{0}=\int_{K 3 \times S^{1} \times S^{2}} H_{7}$,

where $H_{7}=e^{-\phi} \tilde{H}_{3}, H_{3}$ is the field strength of the two-form antisymmetric tensor field and $\phi$ is the ten-dimensional dilaton. Here the string is not wrapped around the $S^{1}$ and so remains a string in $D=5$. The corresponding classical solution is simply the fundamental string in $D=5$. This state is associated with the $B_{\mu \nu}$ field and is dual to a vector in $D=5$. The string theory also possesses a perturbative electrically charged pointlike $H$-monopole state (dual to the magnetically charged $H$-monopole state of [25) with charge

$\tilde{e}_{1}=\int_{K 3 \times S^{3}} H_{7}$.

In this case, the string is wrapped around the $S^{1}$ and becomes a point in $D=5$. This state is associated with the $B_{\mu 5}$ field, where $x_{5}$ is the direction compactified on the $S^{1}$. The $T$-dual electrically charged point-like Kaluza-Klein state has charge $\tilde{e}_{2}$ associated with the $g_{\mu 5}$ field. In this case, the corresponding electrically charged solution is given by the extremal Kaluza-Klein black hole solution of heterotic string theory [26]. The fundamental string state can be identified with one of the three states arising from the Mtheory fivebrane by setting $m_{0}=\tilde{m}_{0}$, while the $H$-monopole and Kaluza-Klein states can be identified with two of the three states arising from the M-theory membrane by setting $e_{1}=\tilde{e}_{1}$ and $e_{2}=\tilde{e}_{2}$. The masses of the identified M-theory and heterotic solutions/states also agree, whether 
computed from central charge/supergravity considerations or from the ADM mass of the classical solutions [24].

The dual case is similar: the heterotic fivebrane wrapped around $K 3 \times S^{1}$ has the nonperturbative (from the string point of view) point-like state with charge

$\tilde{e}_{0}=\int_{S^{3}} H_{3}$

Here the classical solution is simply the heterotic fivebrane wrapped around $K 3 \times S^{1}$, and which is dual to the fundamental heterotic string. One also gets from the heterotic fivebrane a nonperturbative magnetically charged string-like $H$ monopole state with charge

$\tilde{m}_{1}=\int_{S^{1} \times S^{2}} H_{3}$,

where in this case the fivebrane is wrapped around the $K 3$ but reduced on the $S^{1}$. The solution in this case is the usual magnetically charged $H$-monopole (under the field $B_{\mu 5}$ ) 25], which in $D=5$ is a string. The $T$-dual magnetically charged string-like Kaluza-Klein state has charge $\tilde{m}_{2}$ under the field $g_{\mu 5}$. The point-like state can be identified with one of the three states arising from the M-theory membrane by setting $e_{0}=\tilde{e}_{0}$, while the string-like $H$-monopole and Kaluza-Klein states can be identified with two of the three states arising from the M-theory fivebrane by setting $m_{1}=\tilde{m}_{1}$ and $m_{2}=\tilde{m}_{2}$. Again, the M-theory and heterotic masses agree as well.

Note that each of the three pairs of electric/magnetic dual states obey Dirac quantization conditions. Note also that neither the membrane nor the fivebrane from M-theory is in itself sufficient to reproduce the perturbative spectrum of either the five-dimensional string or the dual five-dimensional point-like object. This becomes clear when one realizes that, from the M-theory side, the membrane wrapped around a two-cycle yields only point-like states, while the fivebrane wrapped around a four-cycle yields only stringlike states. On the other hand, from the heterotic compactification, both the string and point-like theories in $D=5$ contain both string and point- like objects in their perturbative spectra. In particular, it follows that the $D=5$ spectrum of Calabi-Yau string solitons yields the fundamental string states on the heterotic side as well as the nonperturbative heterotic string states obtained by wrapping the heterotic fivebrane on $K 3$.

One-loop calculations provide further evidence for this duality [24]. From anomaly considerations, a five-dimensional string action arises which is chiral on the worldsheet. This is especially interesting, since it implies that M-theory calculations may be carried out in the more familiar setting of string theory. Further reduction to $D=4$ yields dual $N=2$ supersymmetric theories 27].

\section{BOUND STATES AND BLACK HOLE THERMODYNAMICS}

The basic fundamental and solitonic $p$-branes preserve half the spacetime supersymmetries, and arise as extremal limits of more general, nonsupersymmetric black $p$-brane solutions of string theory. It turns out, however, that the lowenergy supergravity equations of motion possess a feature that allows for the immediate construction of composite solutions from the basic ones. Consider, for example, the fivebrane solution of eq. (4). Then the generalized connection $\Omega_{\mu}^{a b}=$ $\omega_{\mu}^{a b}-(1 / 2) H_{\mu}^{a b}$, obtained by adding the threeform field strength as torsion to the standard metric connection in the sigma-model frame, is an instanton 25:28: $\Omega_{\mu}^{a b}=\mp(1 / 2) \epsilon^{a b}{ }_{c d} \Omega_{\mu}^{c d}$. The (anti) self-duality of $\Omega$ imposes a chirality choice on the supersymmetry-generating spinor $\epsilon$ which results in halving the number of supersymmetries preserved.

It it not too difficult to see that, by inserting another, independent instanton, we can construct another solution with half again of the number of supersymmetries preserved 29.

$$
\begin{aligned}
& \phi=\phi_{1}+\phi_{2}, \quad g_{\mu \nu}=\eta_{\mu \nu} \quad \mu, \nu=0,1,(11) \\
& g_{m n}=\quad e^{2 \phi_{1}} \delta_{m n} \quad m, n=2,3,4,5 \text {, } \\
& g_{i j}=\quad e^{2 \phi_{2}} \delta_{i j} \quad i, j=6,7,8,9, \\
& H_{m n p}= \pm 2 \epsilon_{m n p q} \partial^{q} \phi \quad m, n, p, q=2,3,4,5 \text {, } \\
& H_{i j k}= \pm 2 \epsilon_{i j k l} \partial^{k} \phi \quad i, j, k, l=6,7,8,9,
\end{aligned}
$$


with $e^{-2 \phi_{1}} \square_{1} e^{2 \phi_{1}}=e^{-2 \phi_{2}} \square_{1} e^{2 \phi_{2}}=0$, where $\square_{1}$ and $\square_{2}$ are the Laplacians in the (2345) and (6789) spaces, respectively. For constant chiral spinors $\epsilon=\epsilon_{2} \otimes \eta_{4} \otimes \eta_{4}^{\prime}$, this double-instanton string solution solves the supersymmetry equations with zero background fermi fields. Due to the presence of two independent instantons in $\Omega$, the chiralities of the spinors $\epsilon_{2}, \eta_{4}$ and $\eta_{4}^{\prime}$ are correlated by $\left(1 \mp \gamma_{3}\right) \epsilon_{2}=\left(1 \mp \gamma_{5}\right) \eta_{4}=\left(1 \mp \gamma_{5}\right) \eta_{4}^{\prime}=0$, so that $1 / 4$ of the spacetime supersymmetries is preserved.

For either $\phi_{1}=0$ or $\phi_{2}=0$ we recover the solitonic fivebrane, which preserves $1 / 2$ the spacetime supersymmetries, since only one instanton is present. In this respect, this string is the composite of two independent fivebranes intersecting along the string. This feature can in fact be generalized to arbitrary $p$-brane solutions 30 33], whereby a given $p$-brane soliton can be interpreted as the intersection of one or more maximally supersymmetric basic fundamental or solitonic $p$-branes. From the viewpoint of $M$-theory, the statement then translates into saying that all $p$-brane solutions arise as intersections of membranes and fivebranes 30 ].

Compositeness was previously seen in the specific context of string-like solutions of toroidally compactified $D=4$ heterotic string theory [34], where each solution could be understood in terms of four independent harmonic functions. The existence of these latter solutions also pointed to an interesting interplay between supersymmetry and duality. In particular, the different supersymmetry breaking patterns of the string-like solutions conform to the different large duality groups (containing both $S$ and $T$ duality) of the various compactifications.

A related composite picture of $p$-brane solutions is the bound states picture 35 41. For the simplest case of extremal black hole solutions in $D=4$, consider the Einstein-Maxwell-scalar action

$S=\frac{1}{G_{N}} \int d^{4} x\left(R-\frac{1}{2}(\partial \phi)^{2}-\frac{1}{4} e^{-a \phi} F_{2}^{2}\right)$,

where $a$ is an arbitrary parameter. It turns out that for the specific values of $a=\sqrt{3}, 1,1 / \sqrt{3}$ and 0 , supersymmetric extreme black holes arising from string compactifications were found. Moreover, from both the spacetime solutions [36, 37] and supersymmetries [39] points of view, these four solutions can be interpreted as bound states of $1,2,3$ and 4 distinct $a=\sqrt{3}$ black holes, respectively, the latter corresponding to maximally supersymmetric $(N=4$ supersymmetry in an $N=8, D=4$ theory) Kaluza-Klein or $H$ monopole solutions with flat metric on moduli space 42]. For example, the $a=0$ ReissnerNordström black hole arises as a bound state of two $T$-dual pairs of electric/magnetic $a=$ $\sqrt{3}$ black holes, each pair producing an $a=1$ black hole. Again, this feature extend quite naturally to arbitrary supersymmetric $p$-branes in any dimension, as well as to non-extremal, nonsupersymmetric $p$-branes [33].

As we shall see, this compositeness feature is crucial to the recent success of string theory in reproducing the Beckenstein-Hawking 43, 44, formula for the entropy of black holes 45 47. First, however, let us reconsider our quantum gravity discussion from the first lecture. We found that, for an elementary particle to exhibit an event horizon and (possibly) behave like a black hole, it was necessary that its Compton wavelength $\lambda_{C}$ be less than its Schwarzschild radius $R_{S}$. A problem, however, arises in this comparison, because most black holes are thermal objects, and hence cannot reasonably be identified with pure quantum states such as elementary particles. In fact, in accordance with the laws of black hole thermodynamics [43, 44] black holes radiate with a (Hawking) temperature constant over the event horizon and proportional to the surface gravity: $T_{H} \sim \kappa$. Furthermore, black holes possess an entropy $S=A / 4 G_{N}$, where $A$ is the area of the horizon (the area law), and $\delta A \geq 0$ in black hole processes. Finally, the energy law of black hole thermodynamics takes the form $d M=(\kappa / 8 \pi) d A+\Omega_{H} d J$, in analogy with $d E=T d S-P d V$, where in the former case $\Omega_{H}$ is the angular velocity of the horizon and $J$ the angular momentum of the black hole.

Since an elementary particle is a pure state, it has entropy $S=0$. According to the above, then, we need to first consider black holes with zero area. For the specific four-dimensional model 
above, the solutions with $a=\sqrt{3}$ and $a=1$ satisfy this requirement. Furthermore, their quantum numbers (mass and charge) match those of particular supersymmetric states of heterotic string theory [35]. In addition, the dynamics of the black holes agree with the four-point amplitudes of the corresponding string states in the low-velocity limit [48]. For the $a=\sqrt{3}$ black holes and their corresponding states, this scattering is trivial, while for the $a=1$ black holes and their corresponding states, we obtain Rutherford scattering. Of course this quantum number matching and dynamical agreement does not mean we can go ahead and identify the black hole solutions with elementary string states, but at least we know that we may possibly be on the right track.

Let us now consider black holes with $A \neq 0$. These should now correspond to an ensemble of string states. Now the laws of black hole thermodynamics follow from classical general relativity. However, the laws of thermodynamics in general follow from a microcanonical counting of quantum states, i.e., from statistical mechanics. An important test of a theory of quantum gravity is then the following: can one obtain the black hole laws of thermodynamics from a counting of microscopic quantum states, i.e., is there a quantum/statistical mechanical basis for these classical laws? We are interested in performing this test for string theory, where we have already developed a correspondence between $p$-brane solutions and elementary and solitonic states. On the one side, we can construct a black hole solution, compute its area and deduce the entropy from $S=A / 4 G_{N}$. On the other side, we can set up the ensemble of states corresponding to this solution, compute its degeneracy and take the logarithm to obtain the entropy.

Let us focus on a particular solution of the four-dimensional model above, namely the case $a=0$, corresponding to the (extremal) ReissnerNordström black hole. This solution is the bound state of four constituent $a=\sqrt{3}$ black holes. Let us embed this solution in type IIB theory compactified from ten to four dimensions. Then the four charges corresponding to the four constituent black holes are given by (appropriately normal- ized to represent number operators): $Q_{1}$, electric with respect to $B_{\mu \nu}, Q_{5}$, magnetic with respect to $B_{\mu \nu}, N$, electric with respect to $g_{5 \mu}$ and $N^{\prime}$, magnetic with respect to $g_{4 \mu}$, where $x_{4}$ and $x_{5}$ are two compactified directions. $Q_{1}$ represents the charge of a D-string, $Q_{5}$ that of a D-fivebrane and $N$ and $N^{\prime}$ electric and magnetic Kaluza-Klein black holes. Let us also simplify the picture by setting $N^{\prime}=1$.

The classical solution has a nonzero area given by $A=8 \pi G_{N} \sqrt{Q_{1} Q_{5} N}$, hence a BeckensteinHawking entropy $S_{B H}=2 \pi \sqrt{Q_{1} Q_{5} N}$. The setup of the corresponding string states is the following. We are interested in the case of large charges, corresponding to black hole solutions. For large $N$, the number operator $N$ represents the momentum of massless open strings going between $Q_{1}$ D-strings and $Q_{5}$ D-fivebranes. The total number of bosonic modes is then given by $4 Q_{1} Q_{5}$, since there is a degeneracy associated with the extra (6789) part of the compactified space. By supersymmetry, the number of fermionic modes is also $4 Q_{1} Q_{5}$. This system is then like a $1+1$ dimensional gas of massless left moving particles with $4 Q_{1} Q_{5}$ bosonic and fermionic species of particles carrying total energy $N / R$, where $R$ is the radius of the circle. The number of such modes is given by $d(N) \sim \exp 2 \pi \sqrt{Q_{1} Q_{5} N}$, so that $S=\ln d(N)=2 \pi \sqrt{Q_{1} Q_{5} N}=S_{B H}$, in agreement with the area law. This is very exciting, as it is the first time we can derive the area law from a quantum-mechanical theory (string theory).2

This agreement seems to hold for a number of cases. In fact, for a given (static) $p$-brane arising as a bound state or as intersections of basic constituent $p$-branes of charge $Q_{1}, Q_{2}, \ldots, Q_{n}$, the area law for the entropy yields $S=A / 4 G=$ $2 \pi \sqrt{Q_{1} Q_{2} \ldots Q_{n}}$, which again agrees, for large $Q_{i}$, with the microscopic entropy formula obtained by counting string states. This was first seen for five-dimensional extremal black holes in 45] and subsequently for four-dimensional extremal black holes in [46. Analogous results for near-extremal black holes were obtained in [47],

\footnotetext{
${ }^{2}$ This argument is only valid in the large $N$ limit, as otherwise the twisted boundary conditions around the circle have to be taken into account. I am grateful to Juan Maldacena for pointing this out.
} 
which seems to indicate that this sort of factorization is not a property of supersymmetry alone, although it is only for supersymmetric solutions that one can invoke non-renormalization theorems to protect the counting of states in going from the perturbative state-counting picture to the nonperturbative black hole picture.

This last argument is crucial: typically in string theory the Schwarzschild radius $R_{S} \sim l_{P} g^{m}$, while $l_{s} \sim l_{P} g^{-n}$, where $g$ is the string coupling, $l_{P}$ and $l_{s}$ are the Planck and string scale, respectively, and $m$ and $n$ are positive integers (for $D=4$ heterotic string theory, for example, $m=n=1)$. So for $g \ll 1, R_{S} \ll l_{P} \ll l_{s}$, in which case one has a perturbative state (or ensemble) but no black hole to speak of (since the horizon is smaller than the string scale), while for $g \gg 1, R_{S} \gg l_{P} \gg l_{s}$, so that one has a horizon and a black hole, but not a perturbative string state. So unless we have an argument that protects the counting of states in adiabatically tuning the coupling from weak to strong, we have no right to assume that the counting is preserved. Here we rely on nonrenormalization due to suprsymmetry.

The question arises as to whether a similar factorization property holds for arbitrary nonextremal black holes. Such a formula was, in fact, found from the $p$-brane picture in [47, where, however, it was noted that the corresponding Dbrane counting argument was unknown. More recently, it was argued in [49] that the counting arguments relating perturbative states to black holes break down for "fat" black holes, i.e. large black holes far from extremality (such as the Schwarzschild black hole). However, this does not in itself rule out the possibility of an analogous, if not identical, compositeness feature in the general case which will once again recover the Beckenstein-Hawking formula and confirm an important success of string theory as a theory of quantum gravity. Nevertheless, these recent computations (as well the more recent work on greybody radiation [50]) have still not addressed the basic physical question, which is: what is the physical process which takes an ensemble of states into a black hole, and what do the quantum states look like in the black hole (strong coupling) limit? These and other questions remain to be answered, as well as the formulation of a dualitymanifest string theory (M-theory and its other single-alphabet cousins, such as F-theory [51], should be thought of as new names for nonperturbative string theory). In this regard, connections with the better-understood Seiberg-Witten [27] duality seem most promising [52].

\section{REFERENCES}

1. M. B. Green, J. H. Schwarz and E. Witten, Superstring Theory, Cambridge University Press, Cambridge, 1987.

2. A. Giveon, M. Porrati and E. Rabinovici, Phys. Rep. 244 (1994) 77.

3. A. Font, L. Ibáñez and F. Quevedo, Phys. Lett. B249 (1990) 35; J. H. Schwarz and A. Sen, Phys. Lett. B312 (1993) 105; A. Sen, Int. J. Mod. Phys. A9 (1994) 3707.

4. R. Rajaraman, Solitons and Instantons, North-Holland, Amsterdam, 1982.

5. C. Montonen and D. Olive, Phys. Lett B72 (1977) 117.

6. M. J. Duff, R. R. Khuri and J. X. Lu, Phys. Rep. 259 (1995) 213.

7. D. J. Gross, J. A. Harvey, E. Martinec and R. Rohm, Nucl. Phys. B256 (1985) 253.

8. A. Dabholkar, G. W. Gibbons, J. A. Harvey and F. Ruiz Ruiz, Nucl. Phys. B340 (1990) 33.

9. M. J. Duff and J. X. Lu, Nucl. Phys. B354 (1991) 129; ibid. 141; A. Strominger, Nucl. Phys. B343 (1990 )167.

10. M. J. Duff, R. R. Khuri and J. X. Lu, Nucl. Phys. B377 (1992) 281.

11. M. K. Prasad and C. M. Sommerfield, Phys. Rev. Lett. 71 (1975) 760; E. B. Bogomol'nyi Sov. J. Nucl. Phys. 24 (1976) 449.

12. G. T. Horowitz and A. Strominger, Nucl. Phys. B360 (1991) 197.

13. M. J. Duff and J. X. Lu, Nucl. Phys. B416 (1994) 301.

14. M. J. Duff and R. R. Khuri, Nucl. Phys. B411 (1994) 473.

15. C. M. Hull and P. K. Townsend, Nucl. Phys. B438 (1995) 109.

16. E. Witten, Nucl. Phys. B443 (1995) 85; C. 
Vafa and E. Witten, Nucl. Phys. B447 (1995) 261.

17. A. Sen, Nucl. Phys. B450 (1995) 103; J. A. Harvey and A. Strominger, Nucl. Phys. B449 (1995) 535.

18. C. V. Johnson, N. Kaloper, R. R. Khuri and R. C. Myers, Phys. Lett. B368 (1996) 71.

19. J. Polchinski, Phys. Rev. Lett. 75 (1995) 4724.

20. J. Polchinski, S. Chaudhuri and C. V. Johnson, hep-th/9602052.

21. J. H. Schwarz, Phys. Lett. B367 (1996) 97; P. Hořava and E. Witten, Nucl. Phys. B460 (1996) 506.

22. M. J. Duff and K. S. Stelle, Phys. Lett. B253 (1991) 113.

23. R. Güven, Phys. Lett. B276 (1992) 49.

24. I. Antoniadis, S. Ferrara and T. R. Taylor, Nucl. Phys. B460 (1996) 489; S. Ferrara, R. R. Khuri and R. Minasian, Phys. Lett. B375 (1996) 81.

25. R. R. Khuri, Phys. Lett. B259 (1991) 261; Nucl. Phys. B387 (1992) 315.

26. D. J. Gross and M. J. Perry, Nucl. Phys. B226 (1983) 29; R. D. Sorkin, Phys. Rev. Lett. 51 (1983) 87; M. J. Duff, R. R. Khuri, R. Minasian and J. Rahmfeld, Ncul. Phys. B418 (1994) 195.

27. N. Seiberg and E. Witten, Nucl. Phys. B426 (1994) 19; Erratum-ibid.B430 (1994) 485.

28. R. R. Khuri, Phys. Rev. D46 (1992) 4526.

29. R. R. Khuri, Phys. Rev. D48 (1993) 2947.

30. A. A. Tseytlin, Nucl. Phys. B475 (1996) 149; I. R. Klebanov and A. A. Tseytlin, Nucl. Phys. B475 (1996) 179.

31. K. Behrndt, E. Bergshoeff and B.Janssen, Phys. Rev. D55 (1997) 3785; K. Behrndt and E. Bergshoeff, Phys. Lett. B383 (1996) 383.

32. J. P. Gauntlett, D. A. Kastor and J. Traschen, Nucl. Phys. B478 (1996) 544.

33. M. Cvetič and A. A. Tseytlin, Nucl. Phys. B478 (1996) 181.

34. M. J. Duff, S. Ferrara, R. R. Khuri and J. Rahmfeld, Phys. Lett. B356 (1995) 479.

35. M. J. Duff and J. Rahmfeld, Phys. Lett. B345 (1995) 441.

36. M. Cvetič and D. Youm, Phys. Rev. Lett. 75 (1995) 4165; Nucl. Phys. B453 (1995) 259; Phys. Lett. B359 (1995) 87; Phys. Rev. D53
(1996) 584.

37. J. Rahmfeld Phys. Lett. B372 (1996) 198; M. J. Duff and J. Rahmfeld, Nucl. Phys. B481 (1996) 332.

38. E. Witten, Nucl. Phys. B460 (1996) 335.

39. R. R. Khuri and T. Ortin, Nucl. Phys. B467 (1996) 355.

40. N. Khviengia, Z. Khviengia, H. Lu and C. N. Pope, Phys. Lett. B388 (1996) 21; hepth/9605077; H. Lu and C. N. Pope, hepth/9606047.

41. M. Cvetič and A. A. Tseytlin, Phys. Rev. D53 (1996) 5619; Phys. Lett. B366 (1996) 95; A. A. Tseytlin, gr-qc/9608044.

42. R. R. Khuri and R. C. Myers, Nucl. Phys. B466 (1996) 60.

43. J. Beckenstein, Lett. Nuov. Cimento 4 (1972) 737; Phys. Rev. D7 (1973) 2333; Phys. Rev. D9 (1974) 3292.

44. S. Hawking, Nature 248 (1974) 30; Comm. Math. Phys. 43 (1975) 199.

45. C. Vafa and A. Strominger, Phys. Lett. B379 (1996) 99; S. R. Das and S. D. Mathur, Phys. Lett. B375 (1996) 103; C. G. Callan and J. M. Maldacena, Nucl. Phys. B472 (1996) 591.

46. J. M. Maldacena and A. Strominger, Phys. Rev. Lett. 77 (1996) 428; C. V. Johnson, R. R. Khuri and R. C. Myers, Phys. Lett. B378 (1996) 78.

47. G. T. Horowitz, J. M. Maldacena and A. Strominger, Phys. Lett. B383 (1996) 151; G. T. Horowitz, D. A. Lowe and J. M. Maldacena, Phys. Rev. Lett. 77 (1996) 430; J. M. Maldacena, Nucl. Phys. B477 (1996) 168.

48. R. R. Khuri and R. C. Myers, Phys. Rev. D52 (1995) 6988.

49. J. M. Maldacena and L. Susskind, Nucl. Phys. B475 (1996) 679; E. Halyo, A. Rajaraman and L. Susskind, Phys. Lett. B392 (1997) 319.

50. J. Maldacena and A. Strominger, Phys. Rev. D55 (1997) 861; S. S. Gubser and I. R. Klebanov, Phys. Rev. Lett. 77 (1996) 4491; I. R. Klebanov and M. Krasnitz, Phys. Rev. D55 (1997) 3250; I. R. Klebanov and S. D. Mathur, hep-th/9701187; I. R. Klebanov, hep-th/9702076.

51. C. Vafa, Nucl. Phys. B469 (1996) 403.

52. A. Sen, Nucl. Phys. B475 (1996) 562. 